PROCEEDINGS OF THE

AMERICAN MATHEMATICAL SOCIETY

Volume 128, Number 9 , Pages 2561-2568

S 0002-9939(00)05315-6

Article electronically published on February 29, 2000

\title{
MAXIMUM CURVES AND ISOLATED POINTS OF ENTIRE FUNCTIONS
}

\author{
T. F. TYLER
}

(Communicated by Albert Baernstein II)

\begin{abstract}
Given $M(r, f)=\max _{|z|=r}(|f(z)|)$, curves belonging to the set of points $\mathcal{M}=\{z:|f(z)|=M(|z|, f)\}$ were defined by Hardy to be maximum curves. Clunie asked the question as to whether the set $\mathcal{M}$ could also contain isolated points. This paper shows that maximum curves consist of analytic arcs and determines a necessary condition for such curves to intersect. Given two entire functions $f_{1}(z)$ and $f_{2}(z)$, if the maximum curve of $f_{1}(z)$ is the real axis, conditions are found so that the real axis is also a maximum curve for the product function $f_{1}(z) f_{2}(z)$. By means of these results an entire function of infinite order is constructed for which the set $\mathcal{M}$ has an infinite number of isolated points. A polynomial is also constructed with an isolated point.
\end{abstract}

\section{INTRODUCTION}

For the purpose of this paper we shall define a curve to be a simple Jordan arc. Given a transcendental entire function $f(z)$ we can define

$$
M(r, f)=\max _{|z|=r}(|f(z)|) \text { and } \mathcal{M}=\{z:|f(z)|=M(|z|, f)\} .
$$

Clunie [1] (problem *2.49) posed the question as to whether or not the set $\mathcal{M}$ can have isolated points. Following Hardy 2], when points of the set $\mathcal{M}$ lie on all or part of curves, we shall define those parts of the curves as maximum curves. In section 2 we shall show that maximum curves or isolated points are sub-sets of other curves which are locally a finite number of analytic arcs. We shall also find a necessary condition for analytically distinct maximum curves to intersect at a point. We shall describe methods for constructing functions where parts of the maximum curves (including isolated points) lie only on the real axis and in section 4, using these methods, we shall show, by adapting a function of Hardy [2] which is of infinite order, that $\mathcal{M}$ can have an infinite number of such points. In section 4 we shall construct a polynomial having an isolated point.

\section{Maximum CURVes}

Functions of the form $f(z)=c z^{k}(k=0,1,2, \ldots)$ will be excluded from the following arguments as the set $\mathcal{M}$ for these functions comprises the complete complex plane.

Received by the editors June 15, 1998 and, in revised form, October 5, 1998.

2000 Mathematics Subject Classification. Primary 30C80.

Key words and phrases. Maximum curves, isolated points, analytic functions.

(C)2000 American Mathematical Society 
For convenience we define the functions

$$
\begin{gathered}
A_{i}(z)=z \frac{f_{i}^{\prime}(z)}{f_{i}(z)} \text { and } B_{i}(z)=z A_{i}^{\prime}(z), \\
a_{i}(r)=\frac{d\left(\log M\left(r, f_{i}\right)\right)}{d(\log r)} \text { and } b_{i}(r)=\frac{d^{2}\left(\log M\left(r, f_{i}\right)\right)}{d(\log r)^{2}} .
\end{gathered}
$$

We will omit the subscripts when dealing with one function.

We note that, at $z=0, A(z)$ is zero unless $f(0)=0$ and that if $A(z)$ is a constant, then $f(z)$ is of the form $c z^{k}$; this last case we have excluded from our argument.

Using the polar form of the Cauchy-Riemann equations we obtain

$$
A(z)=z \frac{f^{\prime}(z)}{f(z)}=r \frac{\partial}{\partial r} \log \left|f\left(r e^{i \theta}\right)\right|-i \frac{\frac{\partial}{\partial \theta}\left|f\left(r e^{i \theta}\right)\right|}{\left|f\left(r e^{i \theta}\right)\right|} .
$$

Thus at points where $M(r, f)=|f(z)|, A(z)$ is real.

We will call the curves where $\frac{\partial}{\partial \theta}\left|f\left(r e^{i \theta}\right)\right|=0$ and $M(r, f)=|f(z)|$ maximum curves and all curves where $\Im A(z)=0$ beta curves. Those parts of beta curves where $\Re A(z)$ is positive will be called alpha curves.

We also note that if $\theta=\alpha_{i}(r)$ is a maximum curve, then on this curve

$$
\Im A(z)=0 \text { and } \Re A(z)=r \frac{d}{d r} \log \left|f\left(r e^{i \alpha_{i}(r)}\right)\right|=\frac{d(\log M(r, f))}{d(\log r)} .
$$

Hence $\Re A(z)$ is positive on the maximum curve when $r>0$ since $A(0)=0$ and $\log (M(r, f))$ is a convex function of $\log (r)$ by Hadamard's three circles theorem [4]. Thus the maximum curves are a subset of the alpha curves.

In the following lemma we show that if a maximum curve is a part of the real axis, then for $z=x+i y$

$$
B(x)=b(r)=\frac{d^{2}(\log M(r, f))}{d(\log r)^{2}} .
$$

This is also non-negative by Hadamard's three circles theorem.

Lemma 2.1. If in the annulus $0 \leq r_{1} \leq|z| \leq r_{2}$ the positive real axis is a maximum curve of $f(z)$, then $a(r)=A(r)$ and $b(r)=B(r)$ in this annulus. If the negative real axis is a maximum curve, then $a(r)=A(-r)$ and $b(r)=B(-r)$.

Proof. First consider the positive real axis in the annulus $0 \leq r_{1} \leq|z| \leq r_{2}$ where $x=r$. If $f(x)>0$, then by (2.4)

$$
A(x)=x \frac{f^{\prime}(x)}{f(x)}=r \frac{f^{\prime}(r)}{f(r)}=A(r)=r \frac{M^{\prime}(r, f)}{M(r, f)}=\frac{d(\log M(r, f))}{d(\log r)}=a(r) .
$$

If $f(x)<0$, then $M(r, f)=-f(r)$ and consequently $M^{\prime}(r, f)=-f^{\prime}(r)$ so that (2.6) is still true.

On the negative real axis $x=-r$. Then if $f(x)>0$, we have $M(r, f)=f(-r)$ and $M^{\prime}(r, f)=-f^{\prime}(-r)$. If $f(x)<0$, we have $M(r, f)=-f(-r)$ and $M^{\prime}(r, f)=$ $f^{\prime}(-r)$. In both these two cases $x \frac{f^{\prime}(x)}{f(x)}=r \frac{M^{\prime}(r, f)}{M(r, f)}$ so that (2.6) holds when $x=-r$. 
Since $A( \pm r)=a(r)$, then $A^{\prime}( \pm r)= \pm a(r)$ so that $B( \pm r)=r a^{\prime}(r)$. Thus

$$
B( \pm r)=r a^{\prime}(r)=\frac{d^{2}(\log M(r, f))}{d(\log r)^{2}}=b(r) .
$$

Definition 2.1. If $\gamma$ is the range of the function, $z=\alpha(t), t_{0} \leq t \leq t_{1}$, where $\alpha(t)$ is analytic and $\alpha^{\prime}(t) \neq 0$ in this interval, then $\gamma$ is called an analytic curve.

If $f(z)=1+a_{p} z^{p}+a_{p+1} z^{p+1}+\ldots$, Hayman [3] has shown that there are at most $p$ maximum curves passing through the origin and that in the neighbourhood of the origin the beta curves form $2 p$ analytic arcs. The nature of beta curves, away from the origin, is also interesting and following similar arguments we shall show that beta curves are necessarily analytic arcs. We note that the function $A(z)=z \frac{f^{\prime}(z)}{f(z)}$ is analytic, except at points where $f(z)=0$.

First we will consider points $z_{0}$ where $f\left(z_{0}\right) \neq 0$. Suppose that $A\left(z_{0}\right)=w_{0}$ where $w_{0}$ is real, $z_{0} \neq 0$. Then $A(z)-w_{0}$ has a zero of order $p$ at $z_{0}$ so that $A(z)=w_{0}+a_{p}\left(z-z_{0}\right)^{p}+\ldots$ Hence

$$
w=\left(\frac{A(z)-w_{0}}{a_{p}}\right)^{\frac{1}{p}}=\left(z-z_{0}\right)+\ldots
$$

is an analytic function in a neighbourhood of $z_{0}$ with a non-vanishing first derivative. Thus the inverse function $z=\psi(w)$ gives a $(1,1)$ conformal map of a neighbourhood of $w=0$ onto a neighbourhood of $z=z_{0}$. In this map the points where $A(z)$ is real correspond to the images of the $2 p$ rays

$$
\begin{aligned}
w=\left(z-z_{0}\right) & =R e^{i \frac{\pi v-\arg a_{p}}{p}}, v=0,1, \ldots, 2 p-1, \\
& = \pm R e^{i \frac{\pi v-\arg a_{p}}{p}}, v=0,1, \ldots, p-1 .
\end{aligned}
$$

These rays form $p$ analytic arcs $\beta_{\gamma}$, passing through $z_{0}$.

Second if $f(z)$ has a zero at $z=z_{0}\left(z_{0} \neq 0\right)$, then $A(z)$ has a simple pole at $z_{0}$ so that $\frac{1}{A(z)}$ is conformal and there is exactly one beta curve passing through $z_{0}$.

Finally if $f(0)=0$, then $|f(z)|=\left|a_{p}\right|\left|\left(1+\frac{a_{q}}{a_{p}} z^{q-p}+\ldots\right)\right|$. Since $\left|a_{p} z^{p}\right|=\left|a_{p}\right| r^{p}$ is constant on $|z|=r_{0}$, then Hayman's results show that there are $q-p$ beta curves passing through the origin.

We note that if $z_{0}$ lies on a beta curve, then

(i) if $B\left(z_{0}\right) \neq 0, p=1$ and there is only one beta curve,

(ii) if $B\left(z_{0}\right)$ has a zero of order $p-1$, there are $p$ beta curves intersecting at angles $\frac{\pi}{p}$.

We can reformulate (i) as

Theorem 2.1. Let $f(z)$ be an entire function with beta curves defined above. Suppose that there exists a positive $\varepsilon$ such that in the annulus $0<r_{0}-\varepsilon<|z|<r_{0}+\varepsilon$ two beta curves, which are distinct, intersect on the circle $|z|=r_{0}$ at the point $z_{0}=r_{0} e^{i \theta_{0}}$. Suppose further that $\left|f\left(z_{0}\right)\right|>0$. Then

$$
B\left(z_{0}\right)=\left.z \frac{d}{d z}\left\{\frac{f^{\prime}(z)}{f(z)}\right\}\right|_{z=r_{0} e^{i \theta_{0}}}=0 .
$$


Proof. Suppose that $B\left(z_{0}\right) \neq 0$. Then $w=A(z)$ yields a conformal $(1,1)$ map near $z_{0}$. Hence the inverse map $z=A^{-1}(w)$ is also $(1,1)$ conformal at $w_{0}=A\left(z_{0}\right)$ and maps the segment of the real axis through $w_{0}$ onto a single analytic arc. But the set of beta curves near $z_{0}$ consists of more than one arc which contradicts our hypothesis.

Corollary 2.1. If for an entire function $f(z)$ a maximum curve intersects a different beta curve at a point $z_{0}$, then

$$
B\left(z_{0}\right)=\left.z \frac{d}{d z}\left\{\frac{f^{\prime}(z)}{f(z)}\right\}\right|_{z=r_{0} e^{i \theta_{0}}}=0
$$

Corollary 2.2. If for an entire function $f(z)$ two distinct maximum curves intersect at a point $z_{0}$, then

$$
B\left(z_{0}\right)=\left.z \frac{d}{d z}\left\{\frac{f^{\prime}(z)}{f(z)}\right\}\right|_{z=r_{0} e^{i \theta_{0}}}=0
$$

In section 3 we will construct an example which is real on the real axis and is the product of two functions only one of which takes its maximum modulus on the real axis only. If $f(z)=f_{1}(z) f_{2}(z), f(r)=M(r, f)$ and $f_{1}(r)=M\left(r, f_{1}\right)$, then it is evident that $f_{2}(r) \neq 0$ and it will be useful to determine suitable bounds for $\left|f_{1}\left(r e^{i \theta}\right)\right|$ and $\left|f_{2}\left(r e^{i \theta}\right)\right|$ so that the product function $f(z)$ has the property $f(r)=M(r, f)$. If $M(r, f)=f(r)$, then by Lemma 2.1

$$
B(r)=\frac{d^{2} \log (M(r, f))}{d(\log r)^{2}}
$$

which must be non-negative by Hadamard's three circles theorem [4].

Theorem 2.2. Suppose that $f(z)=f_{1}(z) f_{2}(z)$ is the product of two entire functions both of which are real and positive on the positive real axis $0<r_{1} \leq|z| \leq r_{3}$. Suppose further that in this annulus

(i) the maximum of $\left|f_{1}(z)\right|$ occurs only on the positive real axis; and

(ii) $M\left(r, f_{1}\right)>M\left(r, f_{2}\right) \geq \frac{1}{f_{2}(r)}$.

Additionally suppose that there exists a real positive function $\delta(r)$ such that

(iii) in the range $|\theta|<\delta(r), \sup _{|\theta|<\delta(r)} \Re B_{2}\left(r e^{i \theta}\right)<\inf _{|\theta|<\delta(r)} \Re B_{1}\left(r e^{i \theta}\right)$, and

(iv) in the ranges $\delta(r) \leq|\theta| \leq \pi, 2 \log M\left(r, f_{2}\right)<\inf _{\delta(r) \leq|\theta| \leq \pi} \log \left|\frac{M\left(r, f_{1}\right)}{f_{1}\left(r e^{i \theta}\right)}\right|$.

Then in the annulus $r_{1} \leq|z| \leq r_{2}, f(z)$ takes its maximum modulus only on the positive real axis.

Proof. First we shall consider the range $|\theta|<\delta(r)$. If $\log (f(z))=u(r, \theta)+i v(r, \theta)$, then $|f(z)|=e^{u(r, \theta)}$. If, with $r$ fixed, we expand $u(r, \theta)$ as a MacLaurin series we 
obtain

$$
|f(z)|=e^{u(r, 0)+u^{\prime}(r, 0) \theta+u^{\prime \prime}(r, \phi) \frac{\theta^{2}}{2}} \text { where }|\phi|<\delta(r) .
$$

Since $i z \frac{d}{d z}=\frac{d}{d \theta}$, we see that

$$
\begin{aligned}
i z \frac{f^{\prime}(z)}{f(z)} & =i A(z)=\frac{d}{d \theta} u(r, \theta)+i \frac{d}{d \theta} v(r, \theta) \\
\text { and }-B(z) & =\frac{d^{2}}{d \theta^{2}} u(r, \theta)+i \frac{d^{2}}{d \theta^{2}} v(r, \theta) .
\end{aligned}
$$

The functions $A(z), B(z)$ and $f(z)$ are all real on the real axis so that

$$
\begin{gathered}
\left.u(r, \theta)\right|_{\theta=0}=\log f(r),\left.\frac{d}{d \theta} u(r, \theta)\right|_{\theta=0}=-\Im A(r, 0)=0, \\
\quad \text { and }\left.\frac{d^{2}}{d \theta^{2}} u(r, \theta)\right|_{\theta=\phi}=-\Re B(r, \phi) .
\end{gathered}
$$

Hence equation (2.9) reduces to $|f(z)|=f(r) e^{-\Re B(r, \phi) \frac{\theta^{2}}{2}}$. Since $\log (f(z))=$ $\log \left(f_{1}(z)\right)+\log \left(f_{2}(z)\right)$, then $B\left(r e^{i \theta}\right)=B_{1}\left(r e^{i \theta}\right)+B_{2}\left(r e^{i \theta}\right)$ so that by condition (iii) we have

$$
\Re B\left(r e^{i \theta}\right) \geq \Re B_{1}\left(r e^{i \theta}\right)-B_{2}\left(r e^{i \theta}\right)>0 .
$$

Thus, except when $\theta=0|f(z)|<f(r)$ in this sector.

Next we shall consider the regions $\delta(r) \leq|\theta| \leq \pi$. Using first condition (ii) and second condition (iv) we have

$$
\left|\frac{f_{2}\left(r e^{i \theta}\right)}{f_{2}(r)}\right| \leq \frac{M\left(r, f_{2}\right)}{f_{2}(r)} \leq\left(M\left(r, f_{2}\right)\right)^{2}<\frac{M\left(r, f_{1}\right)}{\left|f_{1}\left(r e^{i \theta}\right)\right|}=\frac{f_{1}(r)}{\left|f_{1}\left(r e^{i \theta}\right)\right|}
$$

so that

$$
\left|f\left(r e^{i \theta}\right)\right|=\left|f_{1}\left(r e^{i \theta}\right)\right|\left|f_{2}\left(r e^{i \theta}\right)\right|<f_{1}(r) f_{2}(r)=f(r)
$$

Thus in the annulus, $0<r_{1} \leq|z| \leq r_{2}$,

$$
M(r, f)=|f(z)|
$$

only on the positive real axis.

Corollary 2.3. Suppose that the maximum of $\left|f_{1}(z)\right|$ only occurs on the real axis, that $\log \left(f_{1}(z)\right)=\log \left(f_{1}(-z)\right)$ and that $\log \left(f_{2}(z)\right)=-\log \left(f_{2}(-z)\right)$. If condition (iii) of Theorem 1 is extended to include the range $|\pi-\theta|<\delta(r)$ and the range of condition (iv) is reduced to the range $\delta(r) \leq|\theta| \leq \pi-\delta(r)$, then $M(r, f)=f(r)$ and $M(r, f)=|f(z)|$ only on the real axis.

Proof. Since $\log (f(-z))=\log \left(f_{1}(z)\right)-\log \left(f_{2}(z)\right)$, then in the range $\delta(r) \leq|\theta| \leq$ $\pi-\delta(r)$ we may use the same expansion (2.9) with $B\left(r e^{i \theta}\right)=B_{1}\left(r e^{i \theta}\right)-B_{2}\left(r e^{i \theta}\right)$. The inequality (2.11) follows as before. 


\section{A tRANSCENDENTAL FUNCTION WITH AN INFINITE NUMBER OF ISOLATED POINTS}

Example 3.1. $f(z)=e^{e^{z^{2}}+2 z \sin ^{2} z}$.

In this example

$$
A(r)=2 r^{3} e^{r^{2}}+r(1-\cos 2 r)+2 r^{2} \sin 2 r
$$

and

$$
B(r)=4 r^{2} e^{r^{2}}\left(r^{2}+1\right)+r(2 r-1)(2 r+1) \cos 2 r+6 r^{2} \sin 2 r+r .
$$

We find that, for $r>4$,

$$
B(r)>4 r^{2}\left(r^{2}+1\right)-r\left(4 r^{2}-1\right)-6 r^{2}+r=2 r(r-1)\left(2 r^{2}-1\right)>0 .
$$

We shall now apply Corollary[2.3 to the functions $f_{1}(z)=e^{z^{z^{2}}}$ and $f_{2}(z)=e^{2 z \sin ^{2} z}$ so that $f(z)=f_{1}(z) f_{2}(z)$. We shall show that for $r>4 f(z)$ takes its maximum modulus only on the real axis and that $M(r, f)=e^{e^{r^{2}}+2 r \sin ^{2} r}$ at every point of the positive real axis with isolated points appearing on the negative real axis when $\sin ^{2} r=0$.

First we note that $\log \left(f_{1}(-z)\right)=\log \left(f_{1}(z)\right)$ and $\log \left(f_{2}(-z)\right)=-\log \left(f_{2}(z)\right)$.

(i) $\left|f_{1}(z)\right|=e^{e^{r^{2} \cos 2 \theta} \cos \left(r^{2} \sin 2 \theta\right)}$. Evidently $M\left(r, f_{1}\right)=e^{e^{r^{2}}}$ and this is attained on the positive and negative real axis.

(ii) $\left|f_{2}\left(r e^{i \theta}\right)\right| \leq e^{2 r \sinh ^{2} r}<e^{2 r e^{2 r}}$. Hence, for $r>4$,

$$
M\left(r, f_{1}\right)>M\left(r, f_{2}\right) \geq e^{2 r \sin ^{2} r} \geq 1 \geq \frac{1}{f_{2}(r)} .
$$

(iii) Now

$$
\begin{aligned}
\Re B_{1}\left(r e^{i \theta}\right)= & \Re\left\{4 z^{2}\left(z^{2}+1\right) e^{z^{2}}\right\} \\
= & 4 r^{2} e^{r^{2} \cos 2 \theta}\left\{\left(r^{2} \cos 4 \theta+\cos 2 \theta\right) \cos \left(r^{2} \sin 2 \theta\right)\right. \\
& \left.\quad-\left(r^{2} \sin 4 \theta+\sin 2 \theta\right) \sin (2 \theta)\right\} .
\end{aligned}
$$

Given that $r>4$ we choose $\delta(r)=\frac{\pi}{8 r^{2}}$ so that in the ranges $|\theta| \leq \delta(r)$ and $|\pi-\theta| \leq \delta(r)$ we have $r^{2}|\sin 2 \theta| \leq \frac{\pi}{4}$. Thus $\sin \left(r^{2} \sin 2 \theta\right) \leq \frac{1}{\sqrt{2}} \leq \cos \left(r^{2} \sin 2 \theta\right)$.

We can now simplify the arithmetic by taking the approximations

$$
\delta(r)<\frac{1}{32} \text { and } 2 r^{2} \sin ^{2} \theta<\frac{\pi^{2}}{32 r^{2}}<\frac{2}{100} .
$$

Subsequent further approximations give us, for $\operatorname{such} \theta$,

$$
|\sin 2 \theta|<|\sin 4 \theta|<\frac{1}{8} \text { and } \cos 2 \theta>\cos 4 \theta>\frac{3}{4} .
$$

Hence $r^{2} \cos 4 \theta+\cos 2 \theta>\frac{3 r^{2}}{4}$ and $r^{2} \sin 4 \theta+\sin 2 \theta<\frac{r^{2}}{4}$. Thus

$$
\begin{aligned}
\Re B_{1}(z) & >\frac{4}{\sqrt{2}} r^{2} e^{r^{2}\left(1-2 \sin ^{2} \theta\right)}\left(\frac{3 r^{2}}{4}-\frac{r^{2}}{4}\right)>r^{4} e^{r^{2}}\left(\frac{4}{2 \sqrt{2}} e^{-0.02}\right) \\
& >r^{4} e^{r^{2}} .
\end{aligned}
$$


Now $\Re B_{2}(z)=\Re\left\{z+6 z^{2} \sin 2 z+z\left(4 z^{2}-1\right) \cos 2 z\right\}$ so that we only require an upper bound on the circle $|z|=r$. Thus if $r>4$,

$$
\begin{aligned}
\Re B_{2}(z) & \leq \Re\left\{r+6 r^{2} e^{2 r}+r\left(4 r^{2}-1\right) e^{2 r}\right\}=r e^{2 r}\left(4 r^{2}+6 r+1\right)+r \\
& <12 r^{3} e^{2 r} .
\end{aligned}
$$

Since, for $r>4, \Re B_{1}(z)>r^{4} e^{r^{2}}>12 r^{3} e^{2 r}>\Re B_{2}(z)$, condition (iii) is satisfied.

(iv) In the range $\delta(r) \leq|\theta| \leq \pi-\delta(r)$, with $r>1$,

$$
\begin{aligned}
\log \left|\frac{f_{1}(r)}{f_{1}\left(r e^{i \theta}\right)}\right| & \geq e^{r^{2}}-e^{r^{2} \cos 2 \theta}=e^{r^{2}}\left(1-e^{-2 r^{2} \sin ^{2} \theta}\right) \\
& \geq e^{r^{2}}\left(1-e^{-\frac{8 r^{2} \delta^{2}(r)}{\pi^{2}}}\right)=e^{r^{2}}\left(1-e^{-\frac{1}{8 r^{2}}}\right)>\frac{e^{r^{2}}}{1+8 r^{2}}>\frac{e^{r^{2}}}{9 r^{2}} .
\end{aligned}
$$

Also $2\left|\log \left(\left|f_{2}\left(r e^{i \theta}\right)\right|\right)\right| \leq 4 r \sinh ^{2} r \leq 4 r e^{2 r}$.

Since $\left.\frac{e^{r^{2}-2 r}}{36 r^{2}}\right|_{r=4}>1$ and for $r \geq 4$

$$
\frac{d}{d r}\left(\frac{e^{r^{2}-2 r}}{36 r^{2}}\right)=\frac{e^{r^{2}-2 r}\left(2 r^{2}-2 r-3\right)}{36 r^{2}}=\frac{\left.e^{r^{2}-2 r}\{2(r-2)(r+1)+1)\right\}}{36 r^{2}}>0,
$$

we can deduce that condition (iv) is satisfied for $r \geq 4$.

Thus, by Corollary [2.3] for $r>4|f(z)|$ takes its maximum only on the real axis and hence $f(z)=e^{e^{z^{2}}+2 z \sin ^{2} z}$ has an infinite number of isolated points on the negative real axis.

\section{A polynomial With a Single isolated point}

Investigation of the example in section 3 is made simple as it is of the form $f(z)=e^{g_{1}(z) g_{2}(z)}$ so that we can apply Corollary 2.3 If $f(z)$ is a polynomial, then this corollary becomes much more difficult to use and we shall approach the problem directly.

Example 4.1. $P(z)=C\left(1+z^{2}\right)^{2}+z\left(z^{2}-1\right)^{2}$ where $C>1$.

On the circle $|z|=1$ we find that, with $z=e^{i \theta}$,

$$
\begin{aligned}
|P(z)| \leq C\left|z^{2}\left(z+\frac{1}{z}\right)^{2}\right|+\left|z^{2}\left(z-\frac{1}{z}\right)^{2}\right| & =4 C \cos ^{2} \theta+4 \sin ^{2} \theta \\
& =4 C\left\{1-\left(1-\frac{1}{C}\right) \sin ^{2} \theta\right\} .
\end{aligned}
$$

Since $P(1)=P(-1)=4 C$, then if $C>1,\left|P\left(e^{i \theta}\right)\right|$ takes its maximum at the points \pm 1 . We note that the real axis is a beta curve since $\mathrm{A}(\mathrm{z})$ is real on the real axis and that in the deleted neighbourhood of $r=1, P(r)>P(-r)$. Using Theorem [2.1, since $B(-1)=\frac{2(C-1)}{C}$ and $B(1)=\frac{2(C+1)}{C}$, then with $C>1$, the real axis is the only beta curve passing through $z= \pm 1$ so that the set $\mathcal{M}$ for the polynomial $\mathrm{P}(\mathrm{z})$ has an isolated point at $z=-1$.

Whilst the inequality (4.1) gives an upper bound for $|P(z)|$ on the circle $|z|=1$, it is not the complete picture and in Figure 1 we show the graph of $\left|P\left(e^{i \theta}\right)\right|$ with $C=2$ and $C=0.5$. 


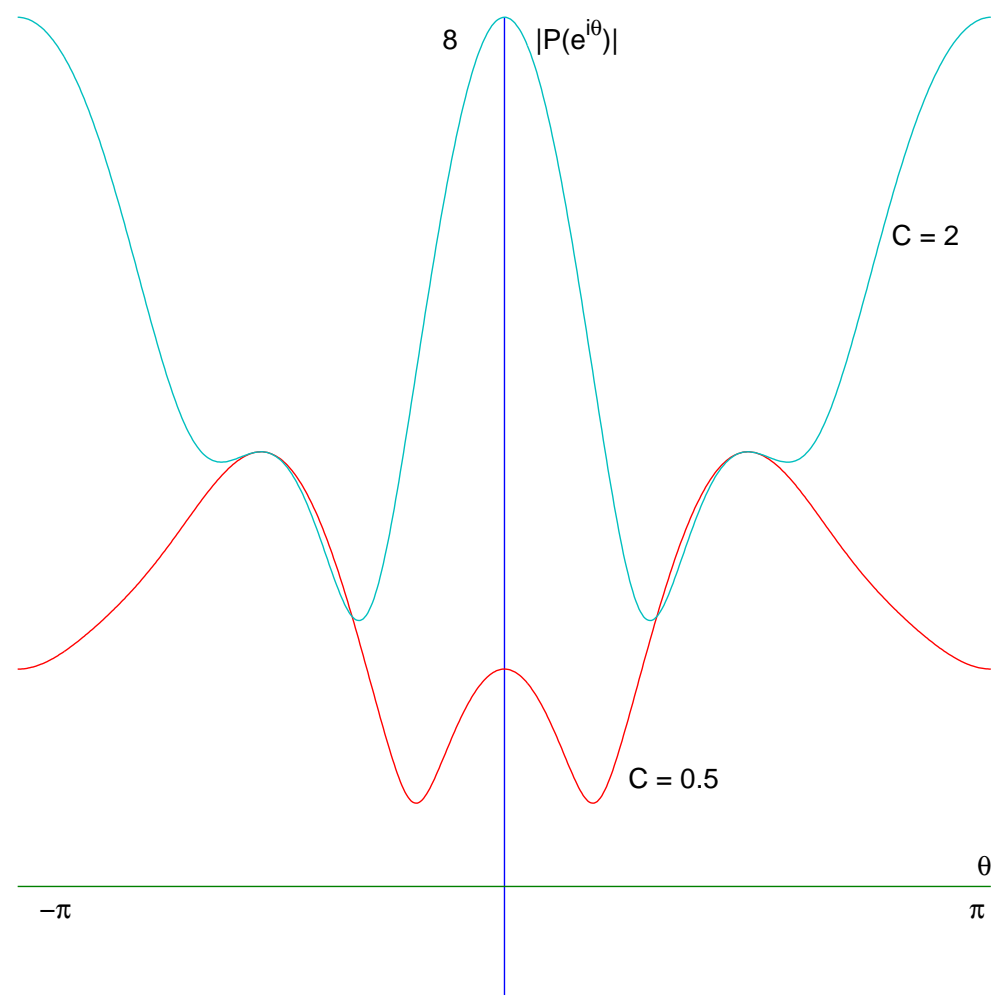

Figure 1. $\left|P\left(e^{i \theta}\right)\right|$

\section{ACKNOWLEDGEMENT}

I wish to thank Professor J. Clunie for suggesting this problem to me and Professor W.K. Hayman for his encouragement and help which forced this work to completion. I would also like to thank Professor A. Eremenko his helpful suggestions and corrections to this paper.

\section{REFERENCES}

[1] J.M. Anderson, K.F. Barth and D.A. Brannan. Research Problems in Complex Analysis. Bull. London Math. Soc. 9 (1977), 129-162. MR 55:12899

[2] G.H. Hardy. "The Maximum Modulus of an Integral Function" . Quart. J.Math. (41) (1909),

[3] W.K. Hayman. A Characterisation, of the Maximum Modulus of Functions Regular at the Origin. J. Analyse Math I (1951) 135-154. MR 13:545d

[4] E.C. Titchmarsh. The Theory of Functions, 2nd ed, Oxford University Press, London, 1939 (reprinted 1975).

University of Greenwich, 30, Park Row, Greenwich, London SE10 9LS, United KingDOM

E-mail address: T.F.Tyler@gre.ac.uk 